whereas no increase was found for radiotherapy.

ANTHONY GREEN

Barnet,
Herts EN5 4QA

1 Chabner B. New Eng $f$ Med 1977 ;297:213-4.

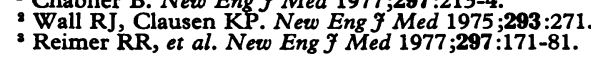

\section{Cervical cancer screening}

SIR, - We were interested to read the opinions expressed in the National Institutes of Health (NIH) consensus statement published in the $B M Y$ (8 November, p 1264) concerning cervical cancer screening policy. There is clearly a degree of uncertainty about the appropriate screening interval (". . . regular intervals of one to three years"), which parallels the American Cancer Society's change of policy from annual to three-yearly screening. ${ }^{1}$

In this country there is debate on the need for regular screening of young women in the face of DHSS policy to reimburse general practitioners only for examinations of women aged 35 or more. ${ }^{23}$ The controversy has been fuelled by statistics showing rising mortality from cancer of the cervix in younger age groups (see accompanying table). In fact, since only a minority of smears are taken by general practitioners most examinations are on women under 35, and have been for many years. 4

Mortality from Cancer of Cervix (per million) England \& Wales

\begin{tabular}{ccc}
\hline & \multicolumn{2}{c}{ Age group } \\
\cline { 2 - 3 } Year & $15-34$ y & Over 34 y \\
\hline 1970 & 6.0 & 170 \\
1971 & 6.6 & 175 \\
1972 & 7.6 & 167 \\
1973 & 6.9 & 170 \\
1974 & 8.2 & 155 \\
1975 & 11.4 & 159 \\
1976 & 10.1 & 165 \\
1977 & 10.5 & 160 \\
1978 & 13.3 & 159 \\
1979 & 00.0 & 000 \\
\hline
\end{tabular}

It should be obvious that, if screening is beneficial at all, the more frequently it is carried out the better the results (cancers prevented, life years saved). However, more intensive programmes involve increasing costs, and there are practical and ethical problems in setting up trials to evaluate the corresponding benefits which might result. Practical difficulties would include the need to compare the effects of a variety of screening regimens on different risk categories of women. Any less comprehensive trials, in which few beneficial effects of screening were detected, would result in calls to repeat the trial with a different pattern of screening or on another risk category of women.

We would support Dr Duncan Neuhauser's comments, in his "minority opinion," concerning the value of using computer-based policy models. These models may be constructed for testing a variety of screening policies on mathematical populations of women. ${ }^{56}$ Ethical difficulties are avoided, and practical problems are reduced to testing the assumptions made in the model about the natural history of the disease and the effects of screening. We have recently constructed our own interactive model, in which a population of women defined by age, parity, and marital status are allowed mathematically to be born, age, give birth, be screened, develop cervical cancer, and die of that disease or from other causes. The influence of screening policies can be simulated; and medical, human, and financial indices may be examined.

Validation of such models is inherently difficult. Existing survey data and consensus statements of the type made by NIH are of value to model builders in providing data against which to validate. Confidence in the predictions of these models comes only with sensitivity analysis. Such models do, however, allow students and planners to experiment with alternative policies and get a feel for the dynamics of the situation, which is simply not possible in the analysis phase of a clinical trial.

The impossibility of carrying out correctly planned clinical trials for screening may not be the great disadvantage one might at first consider it.

D M PARKIN

Leeds Area Health Authority

(Teaching),

A D Clayden

Medical Statistics Section

University Department of

Leeds

1 American Cancer Society. CA-A cancer journal for clinicians $1980 ; 30: 215-23$

2 Anonymous. Lancet 1978; ii:1029-30

- Department of Health and Social Security. Health and personal social services statistics for England. London: HMSO, 1980

- Knox EG. In: McLachlan G, ed. Problems and progress in medical care. Vol 9. London: Oxford Criversity Press, 1973. 122:127-36.

\section{Chasing the unknown primary}

SIR,-With reference to your leading article (8 November, p 1232) on adenocarcinoma from unknown primary sites, several points of clinicopathological importance deserve emphasis.

As you state, the diagnosis of adenocarcinoma must be accurately established and other possible types of tumour excluded. Although not mentioned in your editorial, in difficult cases transmission electron microscopy is a desirable and frequently helpful investigation. In this department electron microscopy of suspected adenocarcinoma has revealed tumours such as poorly differentiated squamous carcinoma, melanoma, apudoma (including oat cell carcinoma), lymphoma, mesothelioma, and malignant histiocytoma. The diagnosis of adenocarcinoma can itself be confirmed by finding one or more characteristic ultrastructural features in the tumour cells. ${ }^{1}$

The importance of establishing the correct diagnosis is so vital that a strong case can be made for establishing NHS centres with a diagnostic electron microscopy service. In Sheffield, we currently offer a "free openaccess" service to surrounding district general hospitals. In most cases an ultrastructural answer is available within 48 hours. A fact not appreciated by many clinicians (and histopathologists) is that the information is frequently obtained from material that has been fixed in formalin or embedded in paraffin wax. Although preservation is not optimal, meaningful answers can be obtained in most instances.

"Special stains" are briefly mentioned in the leading article but their potential importance is not explained. The diagnosis of adenocarcinoma by light microscopy is often dependent on the demonstration of mucin within the tumour cells. Different primary tumours produce different types of mucin and the mucins can be distinguished by relatively simple histochemical techniques. ${ }^{2}$ With this technique, cases of "bronchial" adenocarcinoma have been shown to be secondaries from the large bowel.

The gall bladder, bile duct, and thyroid can be all sites of occult adenocarcinoma but they do not appear in your lists of possible primary sites. Systemic treatment is also said to be warranted for "carcinoma of the testis." Such terminology is presumably derived from the American references quoted. In Britain a different classification of testicular tumours is used $^{3}$ and true adenocarcinoma of the testis is a rarity. To help those who are initiating therapy it should be made clear that you are referring to British subtypes of teratoma.

In the future, most hope for solving a case of adenocarcinoma from an unknown primary site must come from immunoperoxidase techniques. A secretory protein hormone/ enzyme profile will hopefully pinpoint the primary tumour.
primary

\section{Department of Histopathology,}

Royal Hallamshire Hospital
Sheffield S10 2JF

1 Ghadially FN. Diagnostic electron microscopy of
tumours. London: Butterworths, 1980:68-77.
${ }^{2}$ Culling CFA, Reid PE, Burton JD, Dunn WL.
尹 Clin Path 1975;28:656-8.
${ }^{3}$ Pugh RCB. Pathology of the testis. Oxford: Blackwell

Scientific Publications, 1976:139-59.

\section{Pneumonia during treatment of} acute leukaemia

SIR,-Your recent leading article "Pneumonia during the treatment of acute leukaemia," ( 8 November, p 1235) provides an interesting, if selective, summary of experience in certain centres in the USA, but has little relevance to the infective problems encountered in leukaemia units in Britain, and may indeed be misleading if interpreted as a guide to practice here. The most common infections in patients with neutropenia, the proportion with positive microbiology, the percentage with proved bacteraemia, and the nature of the causative organisms vary sharply both from time to time and from centre to centre in Britain and internationally. The early management of infections requires a knowledge of the current pathogenic flora locally in the wards concerned rather than in Baltimore or Houston.

Our experience in Cambridge suggests that while pneumonias are not infrequent they are neither the most common nor the most important infections in patients with neutropenia and leukaemia. Bacteraemias of uncertain origin, possibly most often arising from the gastrointestinal tract, are more common and more serious causes of the dangerous state of circulatory collapse associated with infection by Gram-negative organisms, which you refer to-with somewhat dubious accuracy-as endotoxaemia. The combination of tobramycin and a cephalosporin is not, in fact, established to be more effective than a number of other possible combinations-for example, gentamycin and carbenicillin. Indeed, the EORTC trial report ${ }^{1}$ given as authority for the tobramycin with cephalosporin recommendation did not use this combination of drugs but did show that of 443 infected sites in 396 patients only 56 were pulmonary, and that of 159 bacteraemias 
with source traced only 13 arose from the lungs.

That isolations of Pseudomonas aeruginosa have become less frequent in certain hospitals in the USA does not necessarily imply the same to be true or even likely in hospitals elsewhere-this organism was the second commonest cause of Gram-negative bacteraemia in patients with neutropenia in Addenbrooke's Hospital in the last three years.

Further aspects of this article are open to similar criticism; we do not wish to labour the point, but simply to emphasise the importance for those who treat leukaemia of working in close collaboration with their own microbiological colleagues and establishing patterns of practice in conformity with their own local needs.

F G J HAYHOE J K H REES

Department of Haematological Medicine, University Clinical School,

\section{The EORTC International Antimicrobial Therapy}

SIR,-The recent leading article (8 November, p 1235) on this topic is likely to create misunderstanding about the pattern of infections occurring during treatment of childhood leukaemia. It is implied that the use of methotrexate and the intensification of treatment regimens are recent innovations-as methotrexate has been in use for 30 years and combination chemotherapy with central nervous system prophylaxis for 10 years, neither can surely be classed as such.

The article misinterprets data about the incidence of pneumonia and the time when patients are at risk. The review from the Hospital for Sick Children (HSC) ${ }^{1}$ concerning children diagnosed between 1973 and 1978 related only to infections occurring during remission induction and therefore is not comparable with the report from the Children's Cancer Study Group (CCSG),2 which although it concerned patients treated during the same years calculated the incidence of pneumonia throughout the duration of treatment. The CCSG data clearly show that the peak incidence of pneumonia is not during induction but 40-80 days after diagnosis during continuing (maintenance) therapy. A similar survey of our own patients confirms that pneumonia is an important cause of morbidity and mortality. ${ }^{3}$

Unfortunately more effective antibacterial regimens are unlikely to be of benefit because bacterial pneumonia is not a major cause of death. Treatment of Pneumocystis carini pneumonia with high dose co-trimoxazole has proved effective in our experience ${ }^{4} 5$ The commonest cause of death in remission in patients treated at the HSC is measles pneumonia. Five of 14 deaths in the survey and two additional deaths this year were caused by this infection.

We are concerned at the omission of a strong recommendation for the prevention of measles by immunisation. Live vaccine is, of course, contraindicated in children receiving treatment for leukaemia, but with less than $50 \%$ uptake of measles vaccine in this country a high proportion of children who develop leukaemia have not been immunised. We now recommend that all siblings, and if possible other close contacts of our patients who have not had measles or measles vaccination, should be immunised in an attempt to prevent exposure to infection.

Judith Chessells W C MARSHALI

The Hospital for Sick Children,

The Hospital for Sick
London WCIN $3 \mathrm{JH}$

Chessells JM, Leiper AD. Arch Dis Child 1980; 55:118-23. Siegel SE, Nesbit ME, Baehner R, Sather $\mathrm{H}$, Hammond D. Am f Dis Child 1980;134:28-34. Ninane J, Chessells JM. Arch Dis Child. In Press. Child 1977;52:314-9.

Rapson NT, Cornbleet MA, Chessells JM, Bennett
AJ, Hardisty RM. Br f Haematol 1980;45:41-52.

***The American source cited in the leading article associated the recent increase in the incidence of pneumonia in children with the recent use of intrathecal methotrexate and intensified chemotherapy-ED, $B M \mathcal{F}$.

\section{Granulocytopenia and infections}

SIR,-The passion for supplying granulocytes (leading article, 25 October, p 1091) and for using newer antibiotic regimens (leading article, 8 November, $p$ 1235) in patients who are susceptible to infection should be tempered by the realisation that inadequate defences can be bolstered by provision of specific antibody.

This has been done quite successfully by the use of pneumococcal vaccine and by the use of pseudomonas LPS vaccine. In the case of pseudomonas infections Pollack and Young ${ }^{1}$ showed that antibodies to the exotoxin $\mathrm{A}$ and to lipid-A provide good protection even in patients who are granulocytopenic, and there is much supporting evidence. ${ }^{2} 3$ Protection against all Gram-negative infections (and in addition against meningococcus) would be so much more effective if we organised ourselves so as to provide antilipid $\mathrm{A}$ antibody ${ }^{4}$ or knew which vaccine would effectively do this.

\section{E N WARDLE}

Newcastle upon Tyne NE3 3DE

Pollack M, Young LS. 7 Clin Invest 1979;63:276-86. Kazmierowski J, et al. F Infect Dis 1977;135:438-46. 191-8.

4 Wardle EN. F Clin Path 1980;33:888.

\section{Perinatal and neonatal mortality}

SIR,-Renee Short said of her recent report, ${ }^{1}$ "GPs will not like it, but it's lives that count." She is only partly correct; many GPs will not like it because it's lives that count.

The hypothesis of the working party is that general practitioner obstetrics, other than in units physically attached to consultant units, is unsafe. For this hypothesis no evidence is produced; instead, incomplete evidence to support the opposite conclusion is reported. A number of studies in different parts of the country from GP maternity units, both peripheral and attached, consistently show a perinatal mortality of about one-third of that of consultant units. This is claimed in Mrs Short's report to be due solely to selection of low-risk cases. This is, however, only one more conjecture. To our knowledge, there has been no comparative evaluation of the welfare of a matched group in a consultant unit. In our own peripheral GP maternity unit there has been one only possibly avoidable perinatal death in the last six years, and 2851 deliveries -and these figures include those women

transferred to a consultant unit in labour. In our opinion, there is good reason to believe that selected patients fare better in GP units than in consultant maternity units; for them the latter may be dangerous.

Mrs Short is correct in her assertion that it is lives that count, but her conclusions are not based on evidence, and may therefore be wrong. Let us not arbitrarily close (peripheral) GP maternity units without proper evidence that this is a wise course of action.

E B M HARDING

L T HARRINGTON

C M LOCKWOOD

J R DUNCAN BRown

A C Hall

M A BROWN

N R WHITING

Victoria Hospital,
Lichfield WS13 6QN

${ }^{1}$ Social Services Committee. Perinatal and neonatal mortality. Second report from the Social Services Committee 1979-80. London: HMSO, 1980.

\section{Site of action of intrathecal morphine}

SIR,-May I further comment on Dr J Selwyn Crawford's response (25 October, p 1144) to my previous letter (27 September, p 870)?

It has been demonstrated that opiate receptors exist in the spinal $\operatorname{cord}^{1}$; that opiates introduced intrathecally bind to these receptors; and that, related to this event, analgesia free from the other systemic and cerebral effects of opiates is apparent. Furthermore this can be antagonised by intrathecal naloxone in a dose-dependent fashion. ${ }^{2}$ I trust that this conforms with Dr Crawford's interpretation of "classical 'opiate' activity."

The use of intrathecal ${ }^{3}$ and extradural opiates $^{4}$ in humans has been promising and emphasises the possible relevance of the spinal action of opiates. Furthermore, the observation that intravenous naloxone reverses respiratory depression but not analgesia associated with the administration of intrathecal morphine is another indication of the possible spinal analgesic action. ${ }^{5}$

It has indeed been demonstrated that the human placenta contains two $\beta$-endorphinlike peptides which differ in size from the classical pituitary-derived hormone. ${ }^{6}$ In the same paper ${ }^{6}$ (cited by Dr Crawford), it is mentioned that extracts of liver, lung, thymus, spleen, and kidney were shown to be devoid of $\beta$-endorphin activity. Naturally occurring substances with opiate activity are found mainly in the central nervous system (CNS) and gastrointestinal tract. ${ }^{7}$ Thus Dr Crawford's suggestion that specific receptors for narcotics may be scattered throughout the body appears unfounded in the light of the evidence available. ${ }^{6}$ 7

Naturally occurring opiates may serve as neurotransmitters or modulator substances. ${ }^{8}$ They are capable of producing analgesia. There appear to be multiple opiate receptors in the CNS and gastrointestinal tract with differing characteristics, ${ }^{9}$ and evidence has recently become available that opiate analgesia is mediated by a subpopulation of opiate receptors. ${ }^{10}$ Hence a pattern appears to be emerging with respect to receptor distribution and opiate function which is at variance with Dr Crawford's suggestion that "endorphins may be analogous to prostaglandins."

The laboratory evidence for a selective spinal 